\title{
3D Elastic Registration of Vessel Lumen from IVUS Data on Biplane Angiography
}

\author{
Benoit Godbout ${ }^{1,2}$, Jacques A. de Guise ${ }^{1}$, Gilles Soulez ${ }^{2}$, and Guy Cloutier ${ }^{3}$ \\ ${ }^{1}$ Laboratoire de Recherche en Imagerie et Orthopédie, CRCHUM, ETS \\ 1560 Sherbooke Est (Y-1615), Montréal, H2L-4M1, Canada \\ benoit.godbout@etsmtl.ca \\ http: / /www. lio.etsmtl.ca \\ ${ }^{2}$ Département de Radiologie, CHUM \\ 1560 Sherbooke Est, Montréal, H2L-4M1, Canada \\ ${ }^{3}$ Laboratoire de Biorhéologie et Ultrasonographie Médicale \\ 1560 Sherbooke Est (Y-1619), Montréal, H2L-4M1, Canada \\ http://www.radiol.umontreal.ca/Recherche_6.htm
}

\begin{abstract}
Accurate reconstructions of vessels are important in the understanding of blood flow anomalies. We present a new elastic registration method to reconstruct the lumen of the femoral artery by fusion of Intravascular Ultrasound data and biplane angiography. The catheter path and pullback parameters are found by minimizing width discrepancy in the back-projections without the need for explicit catheter segmentation. Preliminary results on real subjects are promising and show the convergence of the algorithm.
\end{abstract}

\section{Introduction}

The construction of realistic models of arteries is important in the understanding of artery disease and blood flow anomalies. Planar angiograms remain the prevalent imaging technique but there are serious limitations in working with a single planar projection when 3D measurements are needed. The limited spatial resolution and the cost of 3D imaging techniques like CTA, MRA or rotational angiography can also be a serious drawback.

Intravascular Ultrasound (IVUS) is a powerful imaging technique for evaluating the lumen, the vessel wall shape and the plaque composition. By performing a constant speed pullback, one can obtain a reconstruction of a straitened vessel as seen by the tip of the catheter. Without explicit localization of IVUS slices, 3D measurements are distorted.

Some important work on data fusion of IVUS and angiography data has been conducted by several authors $[1,2,3]$. The main research areas are angiographic system calibration, catheter path segmentation, bi-planar curve reconstruction and absolute orientation determination. However, these techniques have some drawbacks when used on clinical data. First, complex calibration objects are not always available. Also, explicit knowledge of the catheter path is mandatory. Angiographic images 
sometimes have poor contrast and, in many cases, segmentation of the catheter can be difficult.

We propose a new 3D elastic registration technique of the lumen model in $2 \mathrm{D}$ angiographic images. A simple calibration technique using a radio-opaque ruler is used. A tubular parametric model of the lumen is constructed from IVUS datasets and the catheter path and absolute orientation are recovered from the projections by an elastic registration of the back-projected lumen model.

We focus our work on the reconstruction of stenoses of the femoral artery. Realistic reconstruction of lumen and vessel wall shapes from patients are essential for our work. Uses will include Computational Fluid Dynamics (CFD) simulation and realistic phantom construction via rapid prototyping technologies.

\section{Method}

The key idea of our method is to register a 3D b-spline tube representing the lumen on the angiograms without explicit knowledge of the pullback start/finish positions and catheter path. We get an estimate of the vessel path from calibrated $2 \mathrm{D}$ views. Using back-projected information, we aim to register this curve to the actual 3D catheter path. Other registration parameters are the pullback endpoints along the vessel and the absolute orientation of the transducer around the catheter. These three degrees of freedom are resolved first. A second step estimates the catheter path by an elastic registration of the lumen model.

\subsection{Calibration of 2D Angiograms}

Two successive angiographic views of the artery at different angles are taken. A radio-opaque ruler fixed to the patient leg gives a series of collinear stereocorresponding points that will be used for calibration. In this study we use a simple linear perspective projection model. Amplifier distortion is considered linear in the central portion of the image. The model could be improved by using more elaborate techniques to compensate non-linear amplifier distortions and errors in the evaluation of acquisition parameters [1].

In our model, geometric parameters including the field of view (FOV), the distance from the x-ray source to the amplifier and the view angles are fixed in the acquisition protocol. The image calibration procedure involves finding the position of the rotation axis of the c-arm in a common 3D reference frame using the back-projected ruler endpoints. The projection matrices are then computed in homogeneous coordinates.

Our angiograms were printed on film and scanned using a VIDAR diagnostic pro film digitizer. For the calibration step, a user must delineate the encompassing circle of both target images and lines of predefined length on the ruler. A typical image is shown in figure 1. 


\subsection{Construction of 3D Lumen Model}

Our IVUS datasets contain about 1000 images acquired during a constant speed pullback. For segmentation purposes, these images are resliced in 32 polar-longitudinal images. Longitudinal contours are extracted using active contours [4] and transformed back into 3D space. An interpolating tubular b-spline surface of degree 3, centered on the catheter, is constructed from these contour points.

The deformation of the parametric b-spline tube $\mathrm{T}(\mathrm{u}, \mathrm{v})$ is controlled by a central $\mathrm{b}$ spline curve $\mathrm{C}(\mathrm{v})$. Control points along the tube are positioned in space using a transform function computed from the control curve. We use the model of relative twist of Whale et al. [2]. This model, built on torsion-free Frenest frames, was validated for small flexion angles and should hold for the femoral artery. The central curve is subdivided in $M$ equidistant points, $M$ being the number of control points of the tube along the axis. The $\mathrm{Z}$ axis of the frame is aligned to the tangent vector to the central curve. An arbitrary normal ( $\mathrm{X}$ axis) is given for the first point and the binormal (Y axis) is computed as the cross product of tangent and normal vectors. The choice of the first normal will impose an absolute orientation of the tube around its central axis. Other normal vectors are computed by rotating, without torsion, the previous frame to align the tangents. Using the computed frames along the central curve, control points of the surface are positioned in 3D space. Figure 2 shows a few frames along the control, curve and the corresponding lumen model.

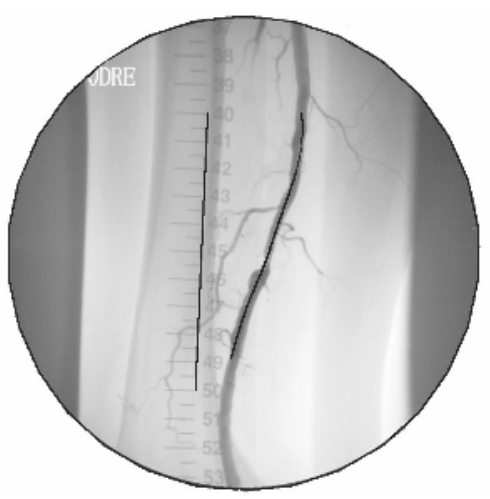

Fig. 1. Initialization process. A user delineates the encompassing circle, the ruler and a few points along the artery.

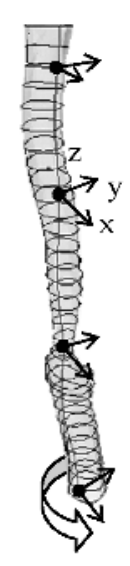

Fig. 2. Deformation of the b-spline tube by frames computed along the central curve starting from an initial absolute orientation.

An estimate of the vessel path is obtained from user supplied points. Using calibration parameters and epipolar constraints, a number of stereo-corresponding points are found along the two $2 \mathrm{D}$ b-splines. These points are reconstructed in $3 \mathrm{D}$ space by 
means of ray intersection. A 3D interpolating b-spline is fitted and the result is used to initialize the central curve for lumen reconstruction. Figure 3 shows the result after initialization. The initial orientation is arbitrary.

\subsection{Absolute Orientation and Position}

The lumen from IVUS data is not cylindrical. We use this fact to better position the tube along the central curve. Stenoses will affect the back-projected width profile along the curve. Asymmetries in the lumen shape will cause variation in the width profile depending on the absolute orientation. The pull-back speed will scale the width profile along the curve. As the $3 \mathrm{D}$ model is back-projected on both angiograms with the calibration matrix, we try to match the resulting width profiles to corresponding widths on the images.

On the model side, we use the initial 3D lumen model to construct width tables for both views. A width table contains values of the back-projected width along the central curve as a function of absolute orientation. The zero crossings of the normal and projection vector dot product are used to extract silhouettes. The width is computed as the back-projected distance between silhouette contours.

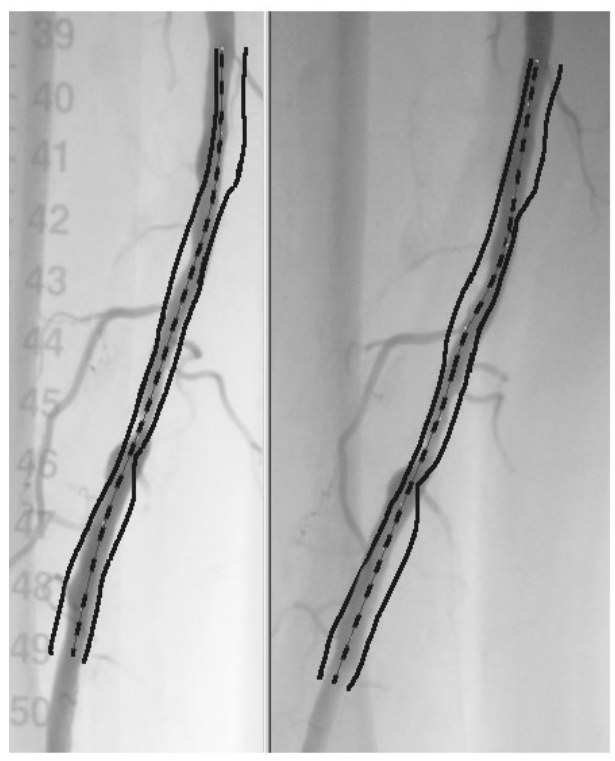

Fig. 3. The initial reconstruction silhouettes are projected on both views.

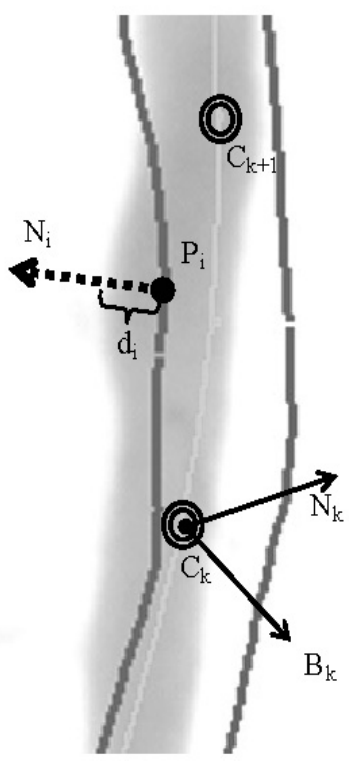

Fig. 4. Elastic registration. The distance $d_{i}$ along the normal $\mathrm{N}_{\mathrm{i}}$ is minimized for every silhouette point $\mathrm{P}_{i}$ by moving the control points $\mathrm{C}_{\mathrm{k}}$ along their normal $\mathrm{N}_{\mathrm{k}}$ and binormal $\mathrm{B}_{\mathrm{k}}$ directions. 
On the image side, the width vectors are defined as the distance between image edges on both sides of the back-projected central curve. We use a smoothing b-spline image to compute the sub-pixel image gradient [5] and search for extrema of the back-projected curve normal and gradient vector dot product.

Translation along the curve and absolute orientation are found by minimizing the least-squared difference of the image width vectors and a subset of the model width tables. The width vectors can be scaled to account for pull-back speed. The new position, orientation and pullback speed are fed to the tube reconstruction to generate a new 3D lumen model.

\subsection{Elastic Registration}

At this stage, we have an estimate of the catheter path start and stop position and absolute orientation. The next step is to refine the catheter path by an elastic registration of the $3 \mathrm{D}$ model on the images.

We search for the gradient maximum in the normal direction along the backprojected silhouettes. As a result, a 3D displacement along the tube normal is generated for every silhouette point on both views.

The centerline displacement $\left(d_{i}\right)$ of a surface point $\left(\vec{P}_{i}\right)$ along its normal $\left(\vec{N}_{i}\right)$ is expressed as a linear equation in the central curve local frame:

$$
d_{i}=\left(\vec{P}_{i} \times \vec{N}_{i}\right)_{t} \cdot r_{o}+\sum \beta_{k}\left(v_{i}\right) \cdot\left[\left(\vec{N}_{k} \cdot \vec{N}_{i}\right) \cdot t_{n, k}+\left(\vec{B}_{k} \cdot \vec{N}_{i}\right) \cdot t_{b, k}\right]
$$

Unknown parameters in the system are translations of the central curve's control points in the normal $\left(\mathrm{t}_{\mathrm{n}, \mathrm{k}}\right)$ and binormal $\left(\mathrm{t}_{\mathrm{b}, \mathrm{k}}\right)$ direction and a small rotation $\left(\mathrm{r}_{\mathrm{o}}\right)$ of the absolute orientation. The weight of each control point is a b-spline function in the central curve parameter space $\left(\beta_{k}\left(v_{i}\right)\right)$. Translation parameters are evaluated in the tube normal direction along the control point normal $\left(\vec{N}_{k}\right)$ and binormal $\left(\vec{B}_{k}\right)$. Figure 4 shows the components of the linear system.

With the solution of this least-squared system, the curve's control points can be translated in 3D space and the absolute orientation updated. The width matching process from section 2.3 and the following elastic registration can be repeated to refine the solution. The resulting deformation can also be applied to other segmented features in the IVUS images like the intima or media borders.

\section{Results and Discussion}

The whole method was integrated into a $\mathrm{C}++$ software developed at our laboratory. The software inputs the bi-plane angiograms and the IVUS contours. It allows interactive reconstruction and visualization of the lumen back-projections. Results are exported as VRML or IGES files to be used as input in CFD, finite element analysis or rapid prototyping software. 
The preliminary validation study is based mostly on visual assessment of the algorithm convergence on real data. The views are taken at 30-degree angle. The vessel model asymmetry appears in the width maps in figure 5 . We see the width values changing with absolute orientation (left to right) and the darker zones correspond to a smaller width near the stenose. Figure 6 shows the registered femoral artery with a stenose. After the elastic registration, the alignment of the stenose and other features seems adequate.

Figure 7 shows an artery after a dilatation procedure. We found the convergence was more dependent on the initial path. It did converge to a satisfying result with good initialization of the catheter start and stop positions.

The whole procedure, including the user delineation of the ruler and initial vessel path, calibration and fine registration takes less than minute to perform. The only parameters are the number of iterations and the control point density. Usually 2 iterations are enough. Results can be manually corrected if a convergence problem should arise.

The choice of control point density will affect the regularity of the final mesh. For our femoral arteries, we use 32 control points around the vessel. Along the axis, the density is proportional to the average perimeter density. These values give us a good regularization of the model while conserving enough precision. The mesh density could be adapted depending on the quality of the initial IVUS segmentation or the final mesh usage. For CFD simulation, a very regular surface is needed and fewer control points should be used.

\section{Conclusion}

We proposed a new elastic registration technique for 3D lumen and artery wall reconstruction from IVUS and bi-plane angiography data. The catheter path and orientation, so as the pullback start and stop positions are found by minimizing width discrepancy in the back-projections. Preliminary results are promising and show the convergence of the algorithm.

Further validation will be conducted. We shall better evaluate the effect of amplifier distortions. Using cross-validation with views taken from different angles will give us a better assessment the 3D path reconstruction quality. Simulated images and images from a realistic phantom could also be used for validation. Full automation is also an important issue as we aim to use these reconstructions in computer-assisted intervention.

Acknowledgement. This work was supported by grants from the Canadian Institute of Health Research (G.C., G.S., \#MOP-53244), Valorisation-Recherche Québec (group grants \#2200-094 and \#2200-003) and NSERC ( J.dG., \#107998-99 ). 


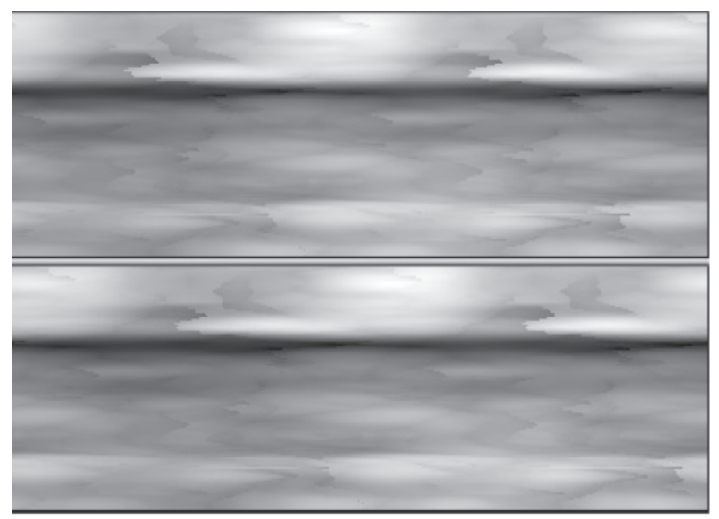

Fig. 5. A graphical view of width tables on $0^{\circ}$ (top) and $30^{\circ}$ (bottom) views. Absolute orientation changes from left to right. The width along the vessel is mapped vertically, white pixels meaning larger width.
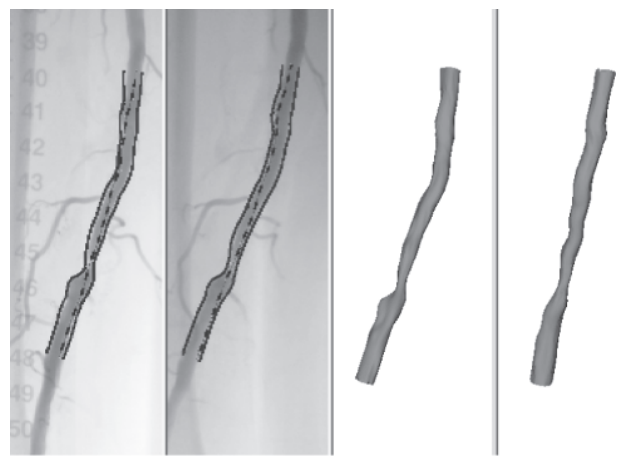

Fig. 6. An example of reconstruction showing a stenose. The catheter path and silhouettes are shown on both views (left). The final 3D reconstruction is shown in frontal and lateral views (right).

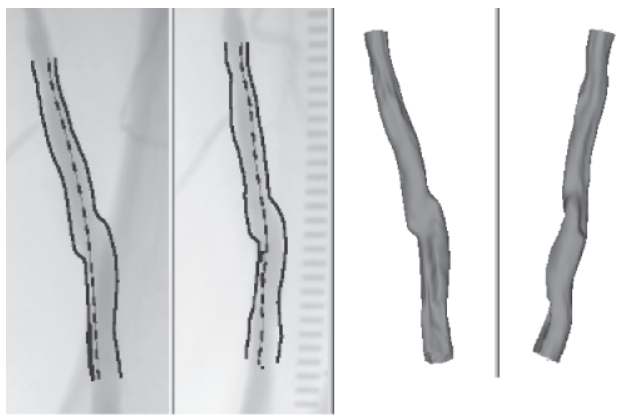

Fig. 7. An example of reconstruction after dilatation. The catheter path and silhouettes are shown on both views (left). The final 3D reconstruction is shown (right). 


\section{References}

1. Cañero, C. , Vilariño, F., Mauri, J. and Radeva, P.: Predictive (un)distortion model and 3D Reconstruction by Biplane Snakes. IEEE Trans. Med. Imag., 19-9 (2002)

2. Wahle, A., Prause, G., DeJong, S. and Sonka, M.: Geometrically correct 3-D reconstruction of intravascular ultrasound images by fusion with bi-plane angiography - methods and validation. IEEE Trans. Med. Imag., 18-8 (1999) 686-99

3. Slager, CJ., Wentzel, JJ., Schuurbiers, JC. et al.: True 3-dimensional reconstruction of coronary arteries in patients by fusion of angiography and IVUS (ANGUS) and its quantitative validation. Circulation. 102-5 (2000) 511-6

4. Godbout, B., Kauffmann, C., de Guise, JA: A simple 2D active contour model to segment non-convex objects in 3D images. Proc. of Vision Interface (VI'98). (1998) 456-64

5. Unser, M., Aldroubi, A., Eden, M. : B-Spline Signal Processing: Part II-Efficient Design and Applications. IEEE Trans. on Signal Processing, 41-2 (1993) 834-48 